\title{
Ionization of one- and three-dimensionally-oriented asymmetric-top molecules by intense circularly polarized femtosecond laser pulses
}

\author{
Jonas L. Hansen, ${ }^{2}$ Lotte Holmegaard, ${ }^{1}$ Line Kalh $ø j,{ }^{1}$ Sofie Louise Kragh, ${ }^{1}$ and Henrik Stapelfeldt ${ }^{1,2, *}$ \\ ${ }^{1}$ Department of Chemistry, Aarhus University, DK-8000 Aarhus C, Denmark \\ ${ }^{2}$ Interdisciplinary Nanoscience Center (iNANO), Aarhus University, DK-8000 Aarhus C, Denmark \\ Frank Filsinger, Gerard Meijer, and Jochen Küpper ${ }^{\dagger}$ \\ Fritz-Haber-Institut der Max-Planck-Gesellschaft, Faradayweg 4-6, D-14195 Berlin, Germany
}

\author{
Darko Dimitrovski, Mahmoud Abu-samha, Christian Per Juul Martiny, and Lars Bojer Madsen ${ }^{\ddagger}$ \\ Lundbeck Foundation Theoretical Center for Quantum System Research, Department of Physics and Astronomy, \\ Aarhus University, DK-8000 Aarhus C, Denmark \\ (Received 8 July 2010; published 7 February 2011)
}

\begin{abstract}
We present a combined experimental and theoretical study on strong-field ionization of a three-dimensionallyoriented asymmetric top molecule, benzonitrile $\left(\mathrm{C}_{7} \mathrm{H}_{5} \mathrm{~N}\right)$, by circularly polarized, nonresonant femtosecond laser pulses. Prior to the interaction with the strong field, the molecules are quantum-state selected using a deflector and three-dimensionally (3D) aligned and oriented adiabatically using an elliptically polarized laser pulse in combination with a static electric field. A characteristic splitting in the molecular frame photoelectron momentum distribution reveals the position of the nodal planes of the molecular orbitals from which ionization occurs. The experimental results are supported by a theoretical tunneling model that includes and quantifies the splitting in the momentum distribution. The focus of the present article is to understand strong-field ionization from 3D-oriented asymmetric top molecules, in particular the suppression of electron emission in nodal planes of molecular orbitals. In the preceding article [Dimitrovski et al., Phys. Rev. A 83, 023405 (2011)] the focus is to understand the strong-field ionization of one-dimensionally-oriented polar molecules, in particular asymmetries in the emission direction of the photoelectrons.
\end{abstract}

DOI: 10.1103/PhysRevA.83.023406

PACS number(s): 33.80.Rv, 33.80.Eh, 42.50.Hz, 37.20.+j

\section{INTRODUCTION}

Photoelectron spectroscopy has been and remains a ubiquitous and useful method to obtain information about the electronic structure of molecules. In the most traditional form, absorption of a single photon frees a single electron from the molecule and the kinetic energy is recorded. The classical use of photoelectron spectroscopy is the determination of binding energies of molecular orbitals and possibly of the vibrational substructure [1]. Over the past 10-15 years such spectroscopy in combination with femtosecond pump-probe methods has been demonstrated to be also a powerful technique for obtaining time-resolved insight into the coupled electronic and vibrational dynamics occurring after photoabsorption in polyatomic molecules $[2,3]$.

In addition to the kinetic energy, the emission direction of the electron is a highly useful experimental observable provided it is detected for defined vibrational and rotational states of the cation formed [4] or if it is measured with respect to the molecular frame $[1,5]$. One experimental approach to obtaining molecular frame photoelectron angular distributions (MFPADs) is to determine the molecular frame after the

\footnotetext{
*henriks@chem.au.dk

†jochen.kuepper@cfel.de; Present address: Center for Free Electron Laser Science, DESY, Notkestrasse 85, 22607 Hamburg, Germany and University of Hamburg, Luruper Chaussee 149, 22761 Hamburg, Germany.

${ }^{\ddagger}$ bojer@phys.au.dk
}

ionization event. This is possible if ionization leads to fragmentation of the molecule and the electrons can be measured in coincidence with fragment ions recoiling in directions that directly reflect the molecular orientation at the moment of ionization, a condition often referred to as the axial recoil limit. Such coincidence methods have been applied successfully, predominantly to small molecules where synchrotron radiation removes an inner shell electron [6-9] but also to situations where valence electrons are removed by UV radiation [10-12].

The other experimental approach to MFPADs is to fix the orientation prior to the ionization event, which offers the obvious advantage that no fragmentation of the molecule is needed. Using resonant photoexcitation mild one-dimensional (1D) alignment can be created for molecules in excited states [5,13]. Alternatively, alignment methods, based on the application of moderately intense, nonresonant laser pulses, can confine the principal axes of polarizability sharply along space-fixed axes [14] and thereby provide the desired fixed-in-space targets for photoionization of molecules possessing an inversion center. The first experiments showing MFPADs measurement from aligned, inversion symmetric (unpolar) linear molecules were reported 2008-2009 [15-17]. Targets of molecules lacking an inversion center (i.e., polar molecules) must, in addition to having their axes confined, possess a directional order of their dipole moment, i. e., the molecules must be oriented as well as aligned [14]. One approach to efficient orientation and alignment is through the combined action of a laser pulse and a weak static electric field [18-21]. 
In the present work we study single ionization of 1Dand 3D-aligned and -oriented samples of the prototypical asymmetric top molecule benzonitrile by intense, circularly polarized femtosecond laser pulses, extending our first results reported recently [22]. For 1D-aligned and -oriented molecules pronounced up-down asymmetries of the electron emission are observed resulting from a higher probability for ionization when the circularly polarized field points along the permament dipole moment of benzonitrile compared to the opposite direction. These results are similar to those obtained for the linear OCS molecules reported in the preceding article [23]. For 3D-aligned and -oriented molecules, striking suppression of electron emission in the polarization plane of the ionizing laser is observed. Our theoretical analysis, relying on a modified tunnel ionization theory, identifies the suppression as resulting from nodal planes in the orbitals from which electrons are removed. Note that effects of nodal plane structure and orbitals below the highest occupied molecular orbital on strong-field ionization of symmetric molecules was discussed theoretically some time ago [24,25].

The article is organized as follows. In Sec. II, the experimental setup is discussed. The experimental results are presented in Sec. III, including a discussion of the method of deflection, alignment, and orientation of the benzonitrile molecules and the MFPADs from strong-field single ionization of benzonitrile by a circularly polarized femtosecond laser field. Section IV contains the theory, including a discussion of the characteristic off-nodal-plane emission pattern. In Sec. V, the theoretical MFPADs are compared with the experimental ones. Section VI concludes and gives a brief outlook. The molecular properties of benzonitrile, used in the theoretical model, are summarized in the Appendix.

\section{EXPERIMENTAL SETUP}

\section{A. Molecular beam apparatus}

An exploded schematic of the molecular beam apparatus, used in these studies, is shown in Fig. 1. It consists of three vacuum chambers: the source chamber, pumped by a $2000-1 / \mathrm{s}$

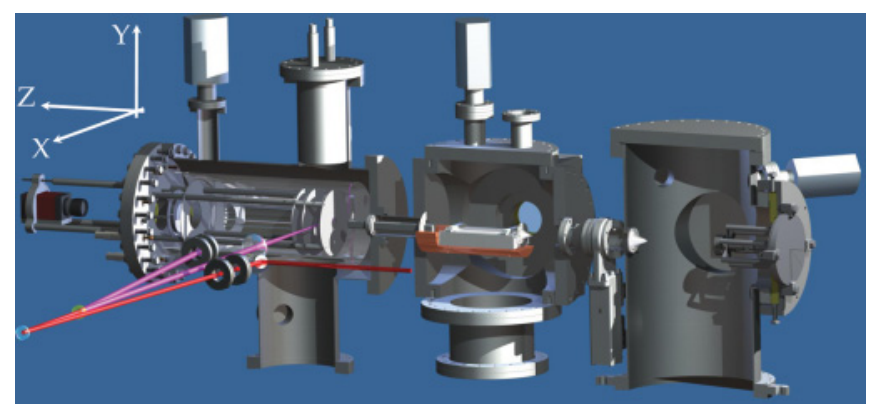

FIG. 1. (Color online) Exploded view of the molecular beam machine consisting of (from right) the source chamber, the deflector chamber, and the target chamber. In this work two laser beams are used, the 1064-nm YAG beam (magenta cylinder) and the 800-nm probe beam (red cylinder). An additional 800-nm femtosecond beam (green cylinder) can be included to induce, for instance, rotational dynamics. The pair of circular disks in each laser beam represents a half-wave and a quarter-wave plate used to control the polarization state of the laser pulses. turbomolecular pump, followed by the deflector and the target chamber which are both pumped by 500-1/s turbomolecular pumps. The molecular beam is formed by expanding a mixture of carrier gas (typically 90 bar of helium) and the molecular gas ( $\sim 5$ mbar of benzonitrile) into the source chamber through a high-pressure Even-Lavie valve [26,27] heated to $40^{\circ} \mathrm{C}$. We estimate the rotational temperature of the molecular gas by (i) comparing experimentally observed degrees of alignment with theoretical calculations for a temperature averaged sample of molecules [28] or (ii) by comparing measured vertical molecular beam profiles of electrostatically deflected molecules with simulations [29]. We find that the typical rotational temperature is $\sim 1 \mathrm{~K}$. The experiments are performed at $20 \mathrm{~Hz}$ limited by the repetition rate of the alignment laser. The molecular beam is skimmed twice, 14 and $38 \mathrm{~cm}$ downstream from the nozzle of the Even-Lavie valve to collimate the expansion. The first skimmer, positioned at the exit of the source chamber, has a 3-mm diameter (Beam Dynamics, model 50.8). In the deflector chamber the molecular beam passes through the second skimmer (1-mm diameter; Beam Dynamics, model 2) just before entering the $15-\mathrm{cm}$-long electrostatic deflector.

\section{B. Electrostatic deflector}

The deflector consists of a trough-shaped electrode with a 3.2-mm inner radius of curvature kept at ground potential and a polished rod with a radius of $3.0 \mathrm{~mm}$ to which high voltage (up to $10 \mathrm{kV}$ ) can be applied [29]. The rod is kept floating above the trough by two pieces of macor, providing a minimum distance of $0.9 \mathrm{~mm}$ between the electrodes and 1.4-mm separation at the molecular beam axis. This electrode geometry creates a strongly inhomogeneous field along the (vertical) $Y$ axis while it is almost homogeneous in the $X$ direction [30]. Therefore, polar molecules traversing the deflector experience a force in the vertical direction that depends on the effective dipole moment of the particular molecular quantum state. As the molecules exit the deflector, they enter a parallel plate capacitor consisting of two 17-cm-long polished stainless steel plates separated by $2.7 \mathrm{~mm}$. The plates are oriented parallel to the deflection direction. The capacitor creates a constant dc electric field on the order of $2 \mathrm{kV} / \mathrm{cm}$ along the path of the molecules moving from the deflector to the laser interaction zone. This finite field prohibits population transfer between quantum states due to Majorana transitions between $M$ levels of individual rotational states at zero field, and it mitigates transfer due to diabatic traversal of avoided crossings induced by rotations or inhomogeneities of the electric field, which occur frequently at small field strengths [31-33].

\section{Velocity map imaging spectrometer and laser beams}

The target chamber houses a velocity map imaging (VMI) spectrometer [34] consisting of an open three-electrode electrostatic lens and a 50-mm-diameter microchannel plate backed by a similar size phosphor screen. Between the first two electrodes (repeller and extractor) the molecular beam is crossed by two pulsed laser beams as indicated with the red and magenta cylinders in Fig. 1. The ion (or electron) images are recorded by a charge-coupled-device camera monitoring the fluorescent phosphor screen, and on-line software analysis determines and saves the coordinates of each individual particle hit. The spectrometer is caged in a single concentric 
mu-metal cylinder to minimize the influence of external magnetic fields on the trajectories of the electrons.

The output from an injection seeded Nd:YAG laser $(20 \mathrm{~Hz}$, $\tau_{\text {FWHM }}=10 \mathrm{~ns}, \lambda=1064 \mathrm{~nm}$ ) is used to adiabatically align the molecules. Part of the output from a $1-\mathrm{kHz}$ amplified Ti:sapphire laser $(800 \mathrm{~nm})$ is compressed to $30 \mathrm{fs}$ and used to either Coulomb explode the molecules (Sec. III B), for detecting their spatial orientation, or to singly ionize the molecules for creating photoelectrons (Sec. III C), or for characterizing the molecular beam profile (Sec. III A). The alignment beam (hereafter termed the YAG beam or the YAG pulse) and the fs beam (hereafter termed the probe beam or the probe pulse) are collinearly overlapped using a zero-degree mirror (high reflectivity at $1064 \mathrm{~nm}$ and high transmission at $800 \mathrm{~nm}$ ) and focused onto the molecular beam by a $50-\mathrm{mm}-$ diameter 30-cm-focal-length spherical lens. The probe pulse is electronically synchronized to the peak of the YAG pulse.

Using telescopes in each laser beam, the spotsizes are adjusted so the probe beam is more tightly focused than the YAG beam $\left(\omega_{0}^{\mathrm{YAG}}=34 \mu \mathrm{m}\right.$ and $\omega_{0}^{\text {probe }}=21 \mu \mathrm{m}$, measured by scanning a $10-\mu \mathrm{m}$ pinhole). The intensity of the probe pulse is adjusted by inserting thin neutral density filters in the beam; the intensity of the YAG pulse is adjusted by rotating a half-wave plate placed between two thin-film polarizers upstream and one thin film polarizer downstream. The polarization state of either beam is controlled by a half-wave and a quarter-wave plate inserted in each beam just before the final zero-degree overlap mirror.

\section{EXPERIMENTAL RESULTS}

\section{A. Deflection of benzonitrile}

The effect of the deflector on the molecular beam is illustrated by the vertical intensity profiles in Fig. 2. These profiles are obtained by recording the $\mathrm{BN}^{+}$signal $[\mathrm{BN}$

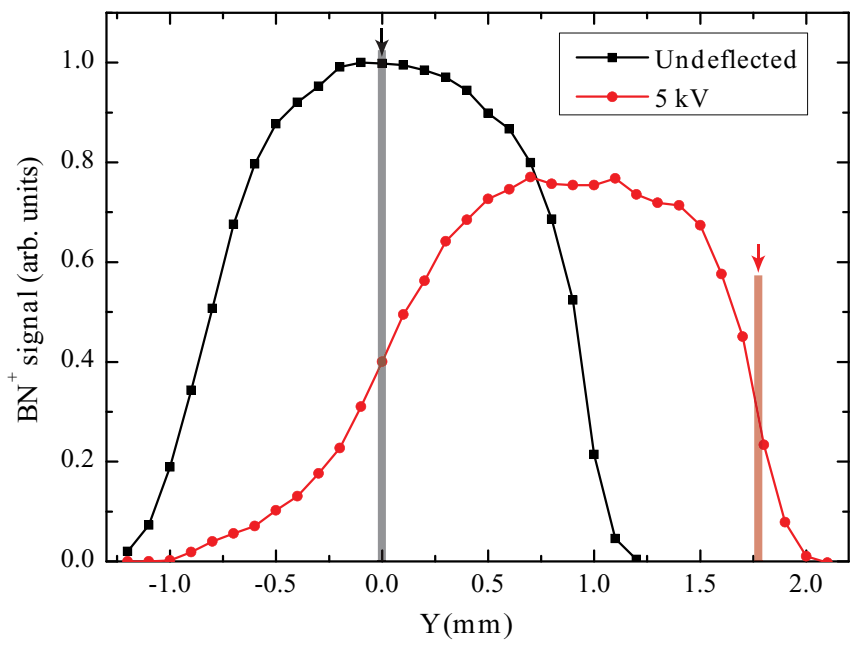

FIG. 2. (Color online) Vertical profiles of the molecular beam measured by recording the $\mathrm{BN}^{+}$signal as a function of the vertical position of the fs probe beam focus. The experimental data are shown by black squares (deflector off, $0 \mathrm{kV}$ ) and red circles $(5 \mathrm{kV})$. The connecting lines serve to guide the eye. The arrows indicate the position of the laser foci for acquiring ion and electron images of undeflected (black), $Y=0 \mathrm{~mm}$, and deflected (red), $Y=1.75 \mathrm{~mm}$, molecules. denotes benzonitrile $\left(\mathrm{C}_{7} \mathrm{H}_{5} \mathrm{~N}\right)$ ] from photoionization due to the femtosecond probe laser $\left(I_{\text {probe }} \approx 10^{14} \mathrm{~W} / \mathrm{cm}^{2}\right)$ as a function of the vertical position of the laser focus. When the deflector is turned off, the molecular beam extends over $\sim 1.7 \mathrm{~mm}$, mainly determined by the diameter of the skimmer before the deflector and the end-aperture of the deflector. When the deflector is turned on, the molecular beam profile broadens and shifts upward (positive $Y$ values). Molecules in the lowest rotational quantum states have the largest Stark shifts and are, therefore, deflected the most [21,29]. In the measurements described below, experiments were conducted on these quantum-state selected molecules simply by positioning the laser foci close to the upper cut-off region in the $5-\mathrm{kV}$ profile $(Y=1.75 \mathrm{~mm})$, as indicated by the red arrow in Fig. 2.

\section{B. Alignment and orientation}

The molecules studied here are asymmetric tops. Their alignment and orientation are controlled by the combination of the YAG pulse and the weak static electric field in the VMI spectrometer. Based on previous experiments and theory, a linearly polarized YAG pulse is used to induce 1D alignment and orientation, whereas 3D alignment and orientation is created by applying an elliptically polarized YAG pulse [14,21,35-38]. Here 1D alignment refers to confinement of a single molecular axis along the YAG polarization axis. Due to the nature of the polarizability interaction between the molecule and the linearly polarized alignment field it is the most polarizable axis that is aligned [14]. For benzonitrile, discussed here, this is the $\mathrm{C}_{2}$ axis (i. e., the C-N axis). Similarly, $3 \mathrm{D}$ alignment refers to confinement of three perpendicular molecular axes [14]. In practice, the elliptically polarized alignment field confines the most polarizable axis along the major polarization axis, the second-most polarizable axis (for $\mathrm{BN}$ the axis in the molecular plane perpendicular to the $\mathrm{C}_{2}$ axis) along the minor polarization axis, and, consequently, the least polarizable axis (for $\mathrm{BN}$ the axis perpendicular to the molecular plane) perpendicular to the polarization plane. Finally, orientation refers to whether the dipole moment of the molecules point in a particular direction with respect to a laboratory-fixed reference direction, which here is the weak static electric field in the VMI spectrometer.

To test that alignment and orientation of BN occurs, the molecules are Coulomb exploded by the intense femtosecond probe pulse $\left(I_{\text {probe }}=5.4 \times 10^{14} \mathrm{~W} / \mathrm{cm}^{2}\right)$ and recoiling ionic fragments are recorded by the $2 \mathrm{D}$ ion detector, a method used previously for many other molecules in our laboratory. For a planar molecule of $C_{2 \mathrm{v}}$ symmetry, like $\mathrm{BN}$, our standard procedure is to first establish that the $\mathrm{C}_{2}$ axis is aligned. This is accomplished by recording $\mathrm{CN}^{+}$ions because they primarily recoil along the $\mathrm{C}_{2}$ axis. Figure $3(\mathrm{~b})$ shows a $\mathrm{CN}^{+}$image recorded with the probe pulse only, linearly polarized in the detector plane. A mild confinement of the ions is observed reflecting the enhanced ionization probability for molecules that initially have their $\mathrm{C}_{2}$ axis pointing along the probe-pulse polarization. The confinement is quantified by $\left\langle\cos ^{2} \theta_{2 \mathrm{D}}\right\rangle=$ 0.63 , where $\theta_{2 \mathrm{D}}$ is the angle between the projection of the $\mathrm{CN}^{+}$ recoil velocity on the detector plane and the YAG polarization [28]. When the YAG pulse is included, linearly polarized vertically, corresponding to $\beta=90^{\circ}$ ( $\beta$ denoting the angle 
(a) Repeller Extractor Ground
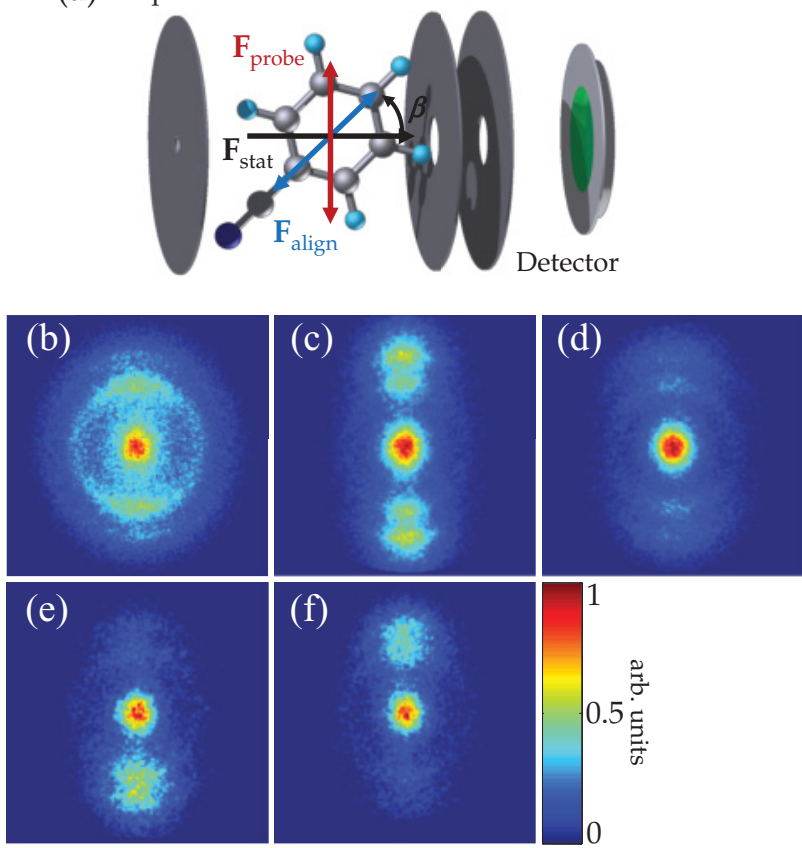

FIG. 3. (Color online) (a) Schematic of the velocity map imaging spectrometer used to detect ions or electrons. The 1D alignment of the $\mathrm{BN}$ molecules is determined by the alignment laser polarization, here shown for $\beta=45^{\circ}$. The static electric field of the spectrometer, pointing from the repeller to the extractor electrode for ion detection, breaks the head-for-tail symmetry by preferentially placing the $\mathrm{CN}$ end toward the repeller where the positive voltage is highest. When detecting electrons the polarity of the electrodes is inverted forcing the $\mathrm{CN}$ end of the molecules toward the extractor electrode. [(b)-(f)] $\mathrm{CN}^{+}$ion images. (b) Probe pulse only, vertically polarized. [(c) and (d)] $\beta=90^{\circ}$. In (d) the rotational temperature is significantly higher than in (c) and no quantum-state selection occurs (see text). [(e) and (f) $\beta=45^{\circ}$ and $-45^{\circ}$, respectively. For all images $\mathbf{F}_{\text {stat }}=286 \mathrm{~V} / \mathrm{cm}$. In (e) [(f)] $28 \%$ [71]\% of all $\mathrm{CN}^{+}$ions appear in the upper half of the detector.

between the YAG polarization and the static electric field), the angular confinement sharpens markedly [Fig. 3(c)] and $\left\langle\cos ^{2} \theta_{2 \mathrm{D}}\right\rangle$ increases to 0.89 . This shows that the $\mathrm{BN}$ molecules are $1 \mathrm{D}$ aligned with their $\mathrm{C}_{2}$ axis along the YAG polarization. The $\left\langle\cos ^{2} \theta_{2 D}\right\rangle$ value is quite a bit smaller than that observed for $1 \mathrm{D}$ alignment of iodobenzene $\left(\left\langle\cos ^{2} \theta_{2 \mathrm{D}}\right\rangle=0.97\right)[21]$ under similar conditions of initial rotational cooling, quantum-state selection, and alignment pulse intensity. This may seem surprising since the components of the polarizability tensor for $\mathrm{BN}$ are similar to those of iodobenzene and thus the two molecules should exhibit approximately the same degree of 1D adiabatic alignment. The reason for the lower $\left\langle\cos ^{2} \theta_{2 D}\right\rangle$ value for $\mathrm{BN}$ is most likely that $\mathrm{CN}^{+}$ions are not ideal observables for deducing the spatial orientation of the $\mathrm{C}_{2}$ axis because, unlike $\mathrm{I}^{+}$ions used in the iodobenzene characterization, it overlaps with other fragment ions in the time-of-flight spectrum of $\mathrm{C}_{7} \mathrm{H}_{5} \mathrm{~N}$. Hence, the ion images of Fig. 3 also contain contributions from hydrocarbon fragments from the benzene ring of equal (or nearly equal) mass-to-charge ratio (e.g., $\mathrm{C}_{2} \mathrm{H}_{2}{ }^{+}$). These "contaminant" ions are not expected to recoil along the $\mathrm{C}-\mathrm{CN}$ axis. In the alignment measurements we try to minimize the contribution from the contaminant ions by only analyzing the $\mathrm{CN}^{+}$ions with the highest kinetic energy corresponding to the outermost ring [39] seen in the images of Fig. 3. However, just a small contributions from the contaminant ions ejected in directions away from the YAG polarization is enough to significantly lower the observed $\left\langle\cos ^{2} \theta_{2 \mathrm{D}}\right\rangle$ value.

For reasons discussed in Sec. IIIC, alignment was also measured for conditions of higher rotational temperature and no rotational state selection. In practice, this is achieved by lowering the He stagnation pressure from 85 to 15 bar [28] and, in addition, turning off the deflector voltages. The pronounced decrease in the degree of alignment is clear by comparing Fig. 3(d) (15 bar He, deflector turned off) with Fig. 3(c) (90 bar He, selection of lowest rotational quantum states). Quantitatively, $\left\langle\cos ^{2} \theta_{2 \mathrm{D}}\right\rangle$ is 0.76 and 0.89 in Figs. 3(d) and 3(c), respectively.

To achieve 3D alignment the polarization of the YAG pulse is changed from linear to elliptical. When an ellipticity of $3: 1$ is chosen, the value referring to the ratio of the intensities measured along the major and the minor polarization axis, the $\mathrm{CN}^{+}$ion image (not shown) recorded with the major polarization axis in the plane of the detector (vertical) is very similar to the image in Fig. 3(c), showing an equally strong degree of $1 \mathrm{D}$ alignment of the $\mathrm{C}_{2}$ axis. To additionally confirm confinement of the molecular plane $\mathrm{H}^{+}$ions were recorded (see Fig. 4) following an approach employed in previous studies $[38,40]$ of $3 \mathrm{D}$ alignment. When the probe laser only is used, the image is circularly symmetric [Fig. 4(a)]. Including the linearly polarized YAG pulse, polarized perpendicular to the detector plane, does not change the circular symmetry [Fig. 4(b)]. This is consistent with the $\mathrm{C}_{2}$ axis being aligned along the YAG polarization vector and the molecular plane free to rotate around the arrested axis. When the 3:1 elliptically polarized YAG pulse, its major polarization axis perpendicular to the detector, is used, the circular symmetry is broken and, instead, the $\mathrm{H}^{+}$ions localize around the vertical axis corresponding to the minor axis of the polarization ellipse. Similarly to previous studies $[38,40]$ we interpret this as confinement of the molecular plane with the second-most polarizable axis (perpendicular to the $\mathrm{C}_{2}$ axis but still in the molecular plane) aligned along the minor axis of the ellipse.
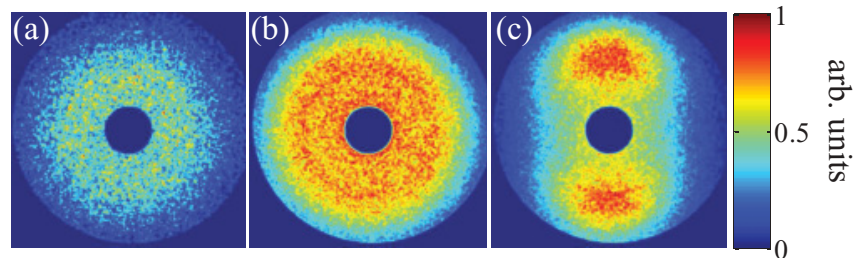

FIG. 4. (Color online) Ion images of $\mathrm{H}^{+}$recorded with the probe laser $\left(5.4 \times 10^{14} \mathrm{~W} / \mathrm{cm}^{2}\right)$ linearly polarized perpendicular to the detector plane. (a) No YAG pulse. (b) YAG pulse included, linearly polarized perpendicular to the detector. (c) YAG pulse included, elliptically polarized. The major axis is perpendicular to the detector and the ellipticity-intensity ratio is $3: 1$. The center has been removed to suppress a large spike of very low kinetic energy $\mathrm{H}^{+}$ions stemming from ionization of residual molecules in the target chamber. 
To show orientation of $\mathrm{BN}$ the polarization of the linearly polarized YAG pulse is rotated to $\beta=45^{\circ}\left(-45^{\circ}\right)$, meaning that the $\mathrm{C}_{2}$ axis of the molecules is positioned at $45^{\circ}\left(-45^{\circ}\right)$ with respect to the static electric field, $\mathbf{F}_{\text {stat }}$, of the VMI spectrometer [see Fig. 3(a)]. The resulting $\mathrm{CN}^{+}$images are displayed in Figs. 3(e) and 3(f)). The appearance of more $\mathrm{CN}^{+}$ions on the lower (upper) part of the detector for $45^{\circ}$ $\left(-45^{\circ}\right)$ shows that the molecules are oriented with the $\mathrm{CN}$ end preferentially pointing toward the repeller electrode. Hence, the permanent dipole moment of $\mathrm{BN}$ is pointing in the direction from the repeller to the extractor as expected. The up-down asymmetry remains unchanged when an elliptically, rather than a linearly, polarized YAG pulse is employed to align the molecules. These findings of alignment and orientation are fully consistent with recent studies on iodobenzene [21,29] and 2,6-difluoroiodobenzene [38].

The degree of orientation is characterized by the ratio of ions detected on the upper part to the total number of ions detected. For $\beta=45^{\circ}$ it is $28 \%$ and for $\beta=-45^{\circ}$ it is $71 \%$. For several reasons these numbers underestimate the degree of orientation utilized in the photoelectron experiments described below. The presence of contaminant ions with the same mass-to-charge ratio as $\mathrm{CN}^{+}$will reduce the up-down asymmetry. In addition, the ion images are recorded at $\mathbf{F}_{\text {stat }}=$ $286 \mathrm{~V} / \mathrm{cm}$, which is significantly lower than the electrostatic extraction field in the photoelectron angular distribution (PAD) measurements presented in Sec. IIIC $\left(\mathbf{F}_{\text {stat }}=467 \mathrm{~V} / \mathrm{cm}\right)$.
In fact, the increase of the static field will be even larger because the ion measurements are recorded at $\beta=45^{\circ}$, i.e., the effective value of $\mathbf{F}_{\text {stat }}$ is reduced by $\sqrt{2}$. By contrast for the PAD measurements, the alignment field is polarized parallel to $\mathbf{F}_{\text {stat }}$. As a consequence, we estimate that the orientation in the PAD geometry corresponds to at least $80 \%$ of the molecules have their $\mathrm{CN}$ end facing the extractor electrode.

\section{PADs from single ionization of benzonitrile}

For the PAD experiments the same experimental setup as for the ion detection described in Sec. III B is used, but some essential parameters are changed. The polarization state of the 30 -fs probe pulses is changed from linear to circular and the intensity is lowered to $\simeq 1.2 \times 10^{14} \mathrm{~W} / \mathrm{cm}^{2}$, corresponding to a regime where the $\mathrm{BN}$ molecules only undergo single ionization with essentially no fragmentation. Also, the polarization of the alignment pulse is changed such that the major axis is parallel to the static field axis. Furthermore, to extract electrons instead of ions in the PAD measurements the polarity of the velocity map imaging spectrometer is inverted. Hereby, the $\mathrm{BN}$ molecules are confined along the static field axis with the $\mathrm{CN}$ end facing the extractor electrode.

The photoelectron images are shown in Fig. 5. Applying only the probe pulse [Figs. 5(a3) and 5(a4)], the electrons emerge in a stripe parallel to the polarization plane $(Y, Z)$ of the probe pulse for both left and right circularly polarized (LCP
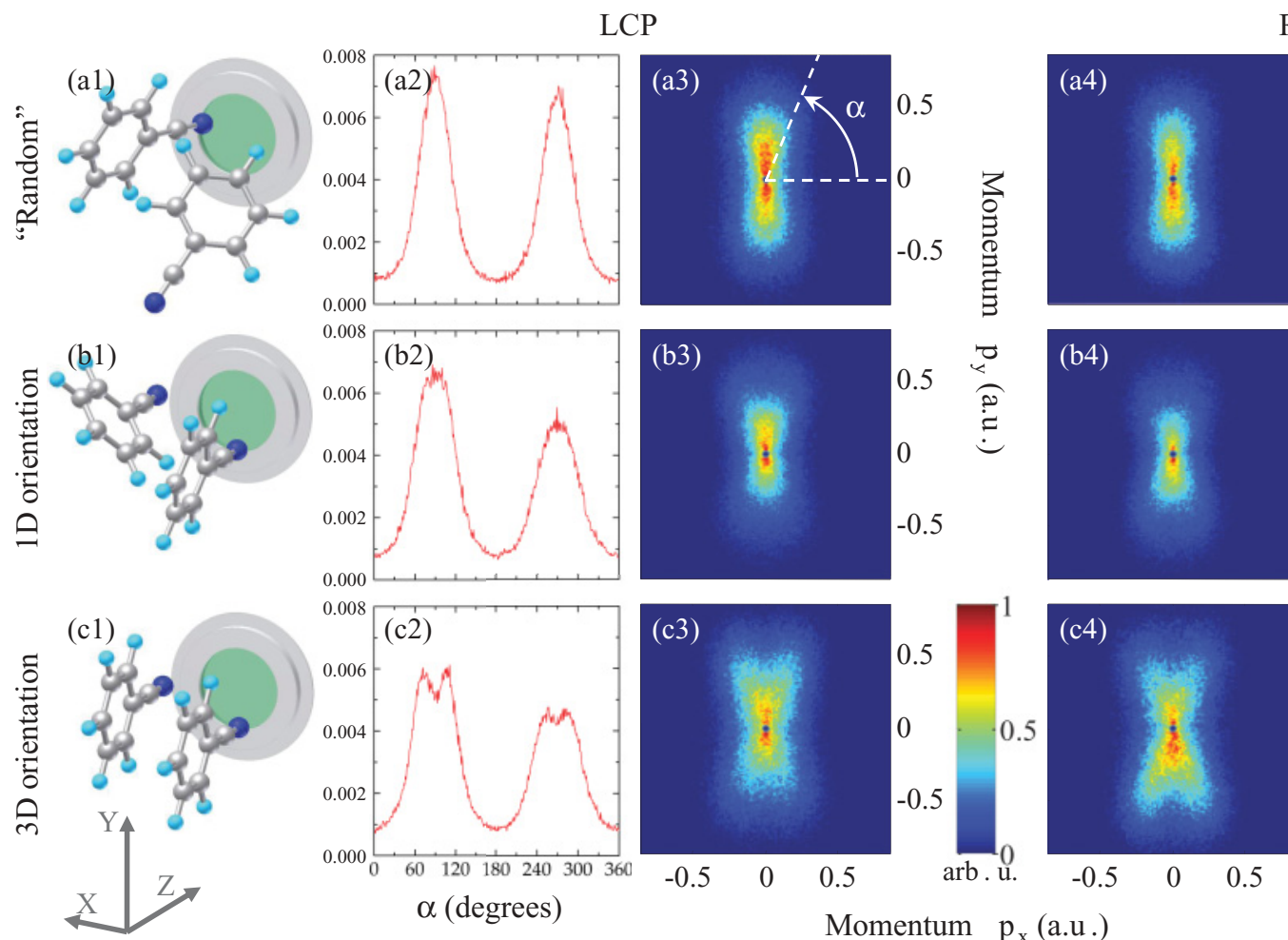

$\mathrm{RCP}$
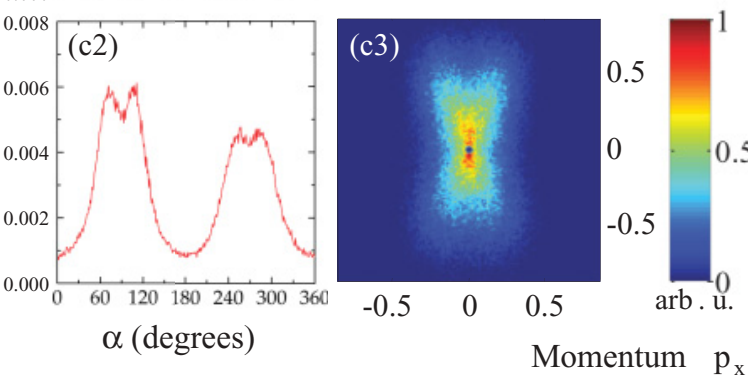

Momentum
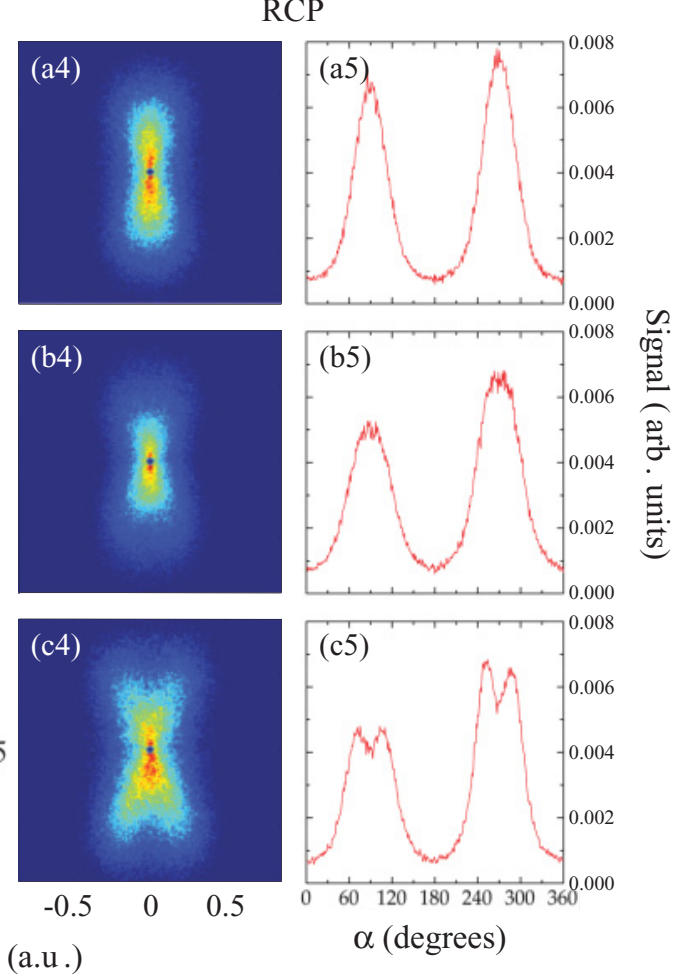

FIG. 5. (Color online) Two-dimensional momentum images of electrons produced when BN molecules are ionized by an LCP probe pulse or an RCP probe pulse. The corresponding angular distributions in the detector plane are shown next to the images. In row (a) the molecules are essentially randomly oriented (no alignment pulse). In row (b) the molecules are 1D aligned and oriented (linearly polarized alignment pulse) and in row (c) the molecules are 3D aligned and oriented (elliptically polarized alignment pulse). The intensity of the probe pulse is $1.2 \times$ $10^{14} \mathrm{~W} / \mathrm{cm}^{2}$. The intensity of the YAG pulse is $7 \times 10^{11} \mathrm{~W} / \mathrm{cm}^{2}$. In row (c) the intensity ratio between the major and minor axis is $3: 1$. The $X, Y, Z$ coordinate system is oriented identical to the one shown in Fig. 1. 


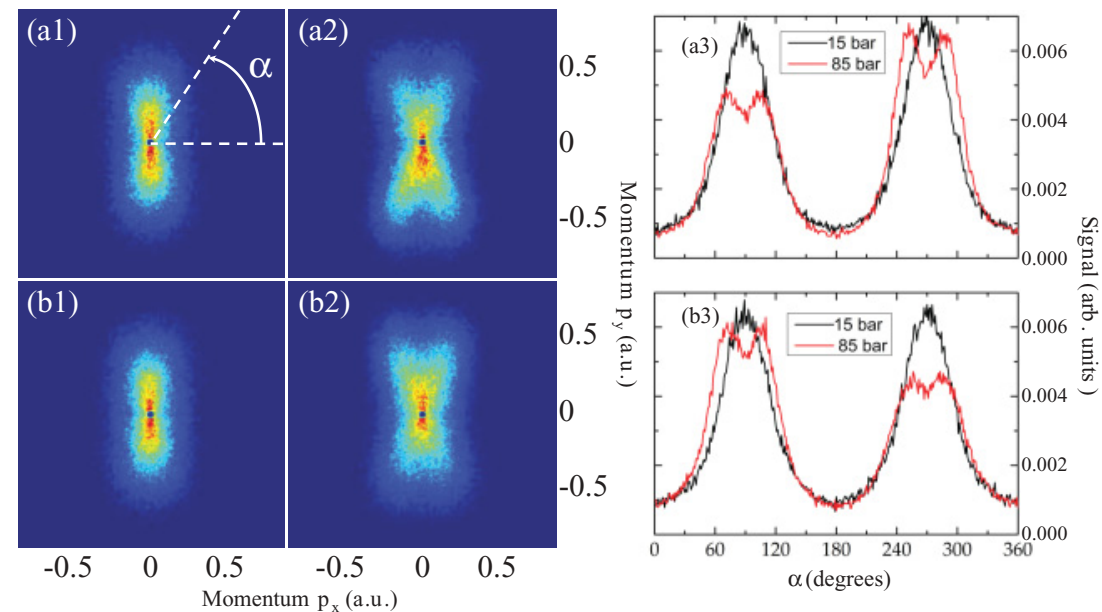

FIG. 6. (Color online) Two-dimensional momentum images of electrons produced when $\mathrm{BN}$ molecules are ionized by a RCP probe pulse [(a1) and (a2)[ or a LCP probe pulse [(b1) and (b2)]. The images in column 1 (2) are recorded for weakly (strongly) 3D-aligned and -oriented molecules obtained by using 15 (85) bar of He stagnation pressure (see text). The laser pulse parameters were the same as those used in Fig. 5. The angular distributions in the detector plane are compared in (a3) and (b3). and RCP) pulses. A weak up-down asymmetry is observed that reverses as the helicity of the probe pulse is flipped. The asymmetry is more easily seen in the angular distributions [Figs. 5(a2) and 5(a5)], obtained by radially integrating the respective images from momentum 0.02 to 0.8 a.u. When the YAG pulse, linearly polarized along $\mathbf{F}_{\text {stat }}$, is included to induce strong 1D alignment and orientation, schematically illustrated in Fig. 5(b1), the up-down asymmetry increases [Figs. 5(b3) and 5(b4)]. For LCP (RCP) probe pulses the number of electrons detected in the upper part compared to the total number in the image is $\sim 55 \%$ (44\%). The enhanced asymmetry is also clearly visible in the angular distributions [Figs. 5(b2) and 5(b5)]. As explained in Sec. IV, the up-down asymmetry results from the orientational dependence of the ionization yield and the force on the electrons, by the probe laser field, following their detachment from the molecule. In particular, we show that ionization of molecules, 1D aligned and oriented with their dipole moment along the direction of $\mathbf{F}_{\text {stat }}$, leads to electrons with an upward (downward) momentum for LCP (RCP) probe pulses in agreement with the observations. The observation of an asymmetry without the YAG pulse results from mild orientation due to the interaction between the permanent dipole moment and the weak static field and/or state selection by the deflector [38].

When the BN molecules are 3D aligned and oriented, obtained by using an elliptically rather than a linearly polarized YAG pulse (see Sec. III B), striking substructures appear in the PADs [Figs. 5(c3) and 5(c4)]. Notably, the electron emission is suppressed along the vertical direction, clearly seen as dips in the corresponding angular distributions [Figs. 5(c2) and 5(c5)] at $\alpha=90^{\circ}$ and $270^{\circ}$, where $\alpha$ is the angle measured counterclockwise with respect to the $X$ direction [see Fig. 5(a3)]. The angular distributions also show that the maximum in the electron emission occurs at an angle

$$
\Omega_{\exp } \sim 18^{\circ}
$$

away from vertical. As explained in Sec. IV the local minimum of electrons along the vertical direction results from suppression of electron emission in the nodal plane of the highest-occupied molecular orbital (HOMO) [and the first orbital below HOMO in energy, HOMO-1] which, for the 3D-aligned and -oriented molecules, corresponds to the
$Y, Z$ plane. Regarding the up-down asymmetry of the electron distributions [Figs. 5(c2) and 5(c5)] it is essentially the same as for the electron distributions obtained for the 1D-aligned and -oriented molecules [Figs. 5(b2) and 5(b5)]. This is in accordance with expectations since the degree of 1D alignment and orientation is essentially the same for the linearly and the elliptically polarized YAG pulse (verified experimentally).

To test that the observed structures in the PADs are not due to perturbation from the YAG pulse on the ionization process by the femtosecond probe pulse, experiments were carried out on molecules with a much lower degree of alignment but identical YAG pulse intensity. This is achieved by employing molecules with higher rotational temperature, achieved as explained in Sec. III B. Figure 6 shows the photoelectron images obtained for LCP and RCP when the molecules are $3 \mathrm{D}$ aligned and oriented. Images similar to those in Fig. 5 are kept as a reference [Figs. 6(a2) and 6(b2)]. Both the up-down asymmetry and the lobelike structures are completely absent in Figs. 6(a1) and 6(b1), thereby showing the importance of a tightly aligned and oriented target for observation of the effects in the PADs. The difference between the experiments on strongly versus weakly aligned and oriented molecules is also obvious from the angular distribution displayed in Figs. 6(a3) and 6(b3).

\section{THEORY}

\section{A. Stark-shift corrected tunneling theory}

To describe the response of molecules with large polarizabilities and dipole moments to strong, near-infrared laser pulses requires the Stark shifts of the energy levels be included; see Ref. [23] and the Supplementary Information in Ref. [22]. In Ref. [23], we modified the tunneling model to include the Stark shifts of the molecule and its cation at the instantaneous value of the external field. We briefly summarize the model here for completeness.

When the Stark shifts are taken into account, the field-free ionization potential $I_{p}(0)$ is modified and reads (up to second order in the field strength $\mathbf{F}$ )

$$
I_{p}(\mathbf{F})=I_{p}(0)+\boldsymbol{\Delta} \boldsymbol{\mu} \cdot \mathbf{F}+\frac{1}{2} \mathbf{F}^{\mathrm{T}} \boldsymbol{\Delta} \boldsymbol{\alpha} \mathbf{F},
$$


where

$$
\Delta \boldsymbol{\mu}=\boldsymbol{\mu}^{M}-\boldsymbol{\mu}^{I} \quad \boldsymbol{\Delta} \boldsymbol{\alpha}=\boldsymbol{\alpha}^{M}-\boldsymbol{\alpha}^{I},
$$

$\boldsymbol{\mu}$ is the permanent dipole moment, $\boldsymbol{\alpha}$ is the polarizability tensor, and the superscripts $M$ and $I$ refer to the molecule and the unrelaxed cation, respectively. We use the unrelaxed cation, since the nuclei have no time to move to the new equilibrium position during the tunneling process. At the field strengths of concern the shifts induced by the hyperpolarizability and higher-order terms are negligible compared to the linear and quadratic terms accounted for in Eq. (2). We note that the dipole moment and polarizability differences in Eq. (3) are exactly the dipole and polarizability of the orbital actively involved in the tunneling process, e.g., the HOMO and HOMO-1 in the present case. Hence the discussion could equivalently be performed referring to the orbital dipole moment and polarizability. Such an approach is natural in the case of calculations with the time-dependent Schrödinger equation within the single-active electron approximation [41].

In the tunneling regime, i. e., when the Keldysh parameter $\gamma$ [42] is smaller than unity, the static Stark shift should be accounted for and the standard tunneling model of Ref. [43] modified accordingly by using the shifted ionization potential of Eq. (2). These changes are crucial in order to reproduce the experimental asymmetry in the photoelectron momentum distribution [22]. First, for simplicity we restrict our consideration to the case were the target molecule can be modeled by an $s$-orbital. We include a factor to account for the saturation of ionization at high intensities [44], and the Stark-corrected tunnel ionization rate (up to a multiplicative constant) becomes

$$
\begin{aligned}
w(\mathbf{F})= & \frac{1}{2 \kappa(\mathbf{F})^{\frac{2}{\kappa(\mathbf{F})}}-1}\left[\frac{2 \kappa(\mathbf{F})^{3}}{F}\right]^{\frac{2}{\kappa(\mathbf{F}}-1} \exp \left[-\frac{2 \kappa(\mathbf{F})^{3}}{3 F}\right] \\
& \times \exp \left\{-6\left[\frac{2}{\kappa(\mathbf{F})^{2}}\right]\left[\frac{F}{\kappa(\mathbf{F})^{3}}\right]\right\},
\end{aligned}
$$

where

$$
\kappa(\mathbf{F})=\sqrt{2 I_{p}(\mathbf{F})} .
$$

We note from Eqs. (2) and (5) that $\kappa(\mathbf{F})$ is a function of the strength and the orientation of the field with respect to the molecular frame.

The Keldysh parameter for the current experiment involving benzonitrile amounts to 1.17. It has been shown that for increasing molecular size the ionization becomes tunnel-like at larger Keldysh parameters [45]; therefore we use the tunneling model here. In benzonitrile the tunnel exit is far from the center of mass, so in circularly polarized laser fields, where no rescattering occurs, the influence of the molecular potential on the escaping electron can be neglected. Accordingly, after the initial ionization step, the subsequent propagation of the electron can be estimated accurately by solving the classical equations of motion in the laser field only [46]. We consider a LCP probe pulse, containing more than 10 cycles, which is switched off adiabatically at large times. These assumptions allow us to consider a field with constant amplitude

$$
\mathbf{F}(t)=F_{0}\left[\sin (\omega t) \hat{\mathbf{e}}+\cos (\omega t) \hat{\mathbf{e}_{\mathbf{z}}}\right]
$$

Here $F_{0}$ is the peak field strength and $\omega$ the angular frequency. The final photoelectron momentum at the $2 \mathrm{D}$ detector screen can now be straightforwardly related to the vector potential at the instant of ionization $t_{0}$, that is

$$
\begin{gathered}
p_{Y}=-A_{Y}\left(t_{0}\right)=-\frac{F_{0}}{\omega} \cos \left(\omega t_{0}\right) ; \\
p_{Z}=-A_{Z}\left(t_{0}\right)=\frac{F_{0}}{\omega} \sin \left(\omega t_{0}\right),
\end{gathered}
$$

where $\omega t_{0}$ is the angle between the $Z$ axis and the field vector at the instant of ionization. Using Eqs. (4) and (7), for each field orientation, i.e., $\omega t_{0}$, the final momenta in the $Y, Z$ plane and the associated tunneling probability can be calculated.

\section{B. Modeling the off-the-nodal plane photoelectron emission by tunneling theory}

The structure of the molecular orbitals of benzonitrile with the lowest binding energies combined with the particular $3 \mathrm{D}$ orientation in the present experimental setup results in the unique $\mathrm{X}$-shaped pattern in the electron momentum distribution shown in Fig. 5, and the off-the-nodal plane emission angle reported in Eq. (1). To explain such a pattern requires a more advanced treatment than the one presented in Ref. [22]. The HOMO and HOMO-1 of benzonitrile are shown in Fig. 7. The most important feature for the present development is that the relevant experimental orientation of the molecule is such that the nodal planes of both the HOMO and HOMO-1 coincide with the molecular plane which in turn coincides with the polarization plane of the laser pulse. In other words, neither HOMO nor HOMO-1 have electron density in the polarization plane of the pulse (6). This geometry poses a theoretical challenge in tunneling models, since conventional tunneling and molecular tunneling theory is inapplicable. The simple reason for their failure is that the standard assumption, that tunneling of the negatively charged electron happens in a very narrow cone around the direction opposite to the field [43,48-50], is no longer valid. In that direction there is no bound state wave function to

\begin{tabular}{|c|c|c|}
\hline Orbital & HOMO & HOMO-1 \\
\hline IP (eV) & 9.79 & 10.06 \\
\hline Shape
\end{tabular}

FIG. 7. (Color online) The HOMO and HOMO-1 of benzonitrile $\left(\mathrm{C}_{7} \mathrm{H}_{5} \mathrm{~N}\right)$. The molecular plane coincides with the $Y, Z$ plane, and the detector is on the right of these images. The insets in dashed lines are end views of the orbitals and show that both orbitals have nodal planes in the polarization plane of the circularly polarized laser pulses. The orbitals and ionization potentials (IP) have been obtained by quantum chemistry calculations in GAMESS [47] at the Hartree-Fock level of theory in conjunction with the valence triple-zeta basis set. 
overlap with the continuum emerging from the outer turning point, and no ionization will occur due to the nodal plane. In short, in the nodal plane there is no population which can tunnel, and consequently emission occur off-the-nodal plane. The simplest cases where this is evident is tunnel emission from a hydrogenic $2 p_{y}$ or $2 p_{x}$ orbital when the field is aligned along the $Z$ axis. These states are linear combinations of the states $|n=2, l=1, m=1\rangle$ and $|n=2, l=1, m=-1\rangle$. According to tunneling theory, the contributions from states with $m=1$ and $m=-1$ should be added incoherently. Then, following Ref. [51], the transverse-momentum distribution is proportional to

$$
\exp \left(-\frac{\sqrt{2 I_{p}}}{F_{0}} p_{X}^{2}\right)
$$

and therefore from such a state one would observe a peak at $p_{X}=0$ in the momentum distributions recorded on the detector screen in this experiment. However, as is evident from the present experiment, the emission occurs off-the-nodal plane. Inclusion of off-the-nodal plane emission in the tunneling model is nontrivial, since the dominant photoelectron momenta under tunneling emission (momentum distribution of tunneled electron at its birth) should now depend on the particular initial state. For example, $2 p_{y}$ or $2 p_{x}$ orbitals would have different transverse-momentum distributions. Concerning the derivation of the tunneling rate, we can, in this situation, no longer separate the azimuthal angle around $\mathbf{F}(t)$ and thus cannot add the individual $m$ components incoherently. We note that even when static Stark shifts are included, the information about the nodal plane of the initial state survives and cannot be washed out by the polarization effects as we will see below.

Motivated by earlier work on tunneling theory for large systems [52], and by the fact that the saddle-point formulation of the length-gauge strong-field approximation (SFA) [42] in the limit of small Keldysh parameter agrees very well with the tunneling theory [53] (at least for a zero-range potential), we modify the transverse-momentum distribution of Eq. (8) by multiplying it by the squared Fourier transform of the initial wave function. More precisely, for the $2 p_{x}$ orbital that means multiplication of the transverse-momentum distribution (8) by the factor $\left(p_{\rho} \cos (\phi)\right)^{2}$, i. e.,

$$
w\left(p_{\rho}, \phi\right) \sim p_{\rho}^{2} \cos ^{2}(\phi) \exp \left[-\frac{\sqrt{2 I_{p}(0)}}{F_{0}} p_{\rho}^{2}\right]
$$

where $p_{\rho}$ and $\phi$ are the two-dimensional polar coordinates in the $X, Y$ plane. In Eq. (9) $\left(p_{\rho} \cos (\phi)\right)^{2}$ is the dominating factor relating to the angular behavior of the squared Fourier transform of $2 p_{x}$ at $p_{Z}=0$, and a factor $\left[(p / \kappa)^{2}+1\right]^{-6}$ has been neglected. Finally, in the above equation for the transverse-momentum distribution, we have used the fieldfree ionization potential (appearing as a square root in the exponential) since, as we have checked, adding Stark shifts to this part of the distribution does not change the results much.

Using the distribution (9) the expectation value of the $X$ component of the transverse momentum (we assume the field is oriented along the $Z$ axis), $p_{X}=p_{\rho} \cos (\phi)$ in the interval $p_{X}>0$ can be calculated analytically as

$$
\left\langle p_{X}\right\rangle=\frac{\int_{0}^{\infty} d p_{\rho} p_{\rho}^{2} \int_{-\pi / 2}^{\pi / 2} d \phi \cos (\phi) w\left(p_{\rho}, \phi\right)}{\int_{0}^{\infty} d p_{\rho} p_{\rho} \int_{-\pi / 2}^{\pi / 2} d \phi w\left(p_{\rho}, \phi\right)}=2 \sqrt{\frac{F_{0}}{\pi \kappa}}
$$

where $\kappa=\sqrt{2 I_{p}(0)}$. We note that the expectation value of $p_{Y}$ for the tunneled electron at birth is $\left\langle p_{Y}\right\rangle=0$ and that the probability of emission with $p_{X}=0$ is zero, i. e., there is no emission in the nodal plane of the initial state although the field is along the $Z$ axis. The final momenta of the tunneled electron along the $Y$ and $Z$ axis are defined by Eq. (7) and it follows that the maximal final electron momentum along the $Y$ direction is $p_{Y}^{\max }=F_{0} / \omega$. We can define an angle $\Omega=\arctan \left(\left\langle p_{X}\right\rangle / p_{Y}^{\max }\right)$ as a measure for the off-the-nodal plane emission. With the above expressions, this angle can be calculated analytically and the results reads

$$
\begin{aligned}
\Omega & =\arctan \left(\left\langle p_{X}\right\rangle / p_{Y}^{\max }\right)=\arctan \left(\frac{2 \omega}{\sqrt{\pi \kappa F_{0}}}\right) \\
& =\arctan \left(\frac{2}{\sqrt{\pi}} \gamma \sqrt{\frac{F_{0}}{\kappa^{3}}}\right),
\end{aligned}
$$

where $\gamma=\omega \kappa / F_{0}$ is the Keldysh parameter [42]. The above expression is, to our knowledge, the first quantification of the angle of off-the-nodal plane emission as a function of the field intensity and frequency and the binding energy of the atomic or molecular system. It has the intuitively correct behavior with respect to increase of the field intensity and the binding energy: The angle decreases. Since both peak field $F_{0}$ and $\kappa$ appear as square roots in the expression, in the limit of large intensities, where also the tunnel model is more valid, the angle is inversely proportional to the fourth root of intensity, i. e., its dependence on intensity is very weak.

To test our model, we imagine a fictitious experiment where we have an "oriented" atom in a $p_{x}$ orbital and the field-free ionization potential $I_{p}(0)=0.5$ a.u.. We assume that, identically to the experiment involving benzonitrile, ionization is induced by a LCP field in the $Y, Z$ plane and that the momentum distribution is recorded on a detector coinciding with the $X, Y$ plane. The final momentum distribution recorded on the screen can be calculated for this case using the above off-the-nodal-plane tunnel emission model. We have compared the results from our model to the calculations within the strong-field approximation [42] assuming a 10-cycle circularly polarized laser pulse with a sine-square envelope, defined in terms of the vector potential $\mathbf{A}(t)$ interacting with the same $2 p_{x}$ state. The comparison between the predictions of $\left\langle p_{X}\right\rangle$ and $\Omega$ by the two methods is given in Fig. 8. It can be seen that for large intensities, the tunneling model and the SFA calculations largely agree for both quantities. As the intensity of the field decreases or, equivalently, the Keldysh parameter $\gamma$ increases, the discrepancy between the SFA and the tunneling model results increases, reflecting the gradual departure from the tunneling regime. 

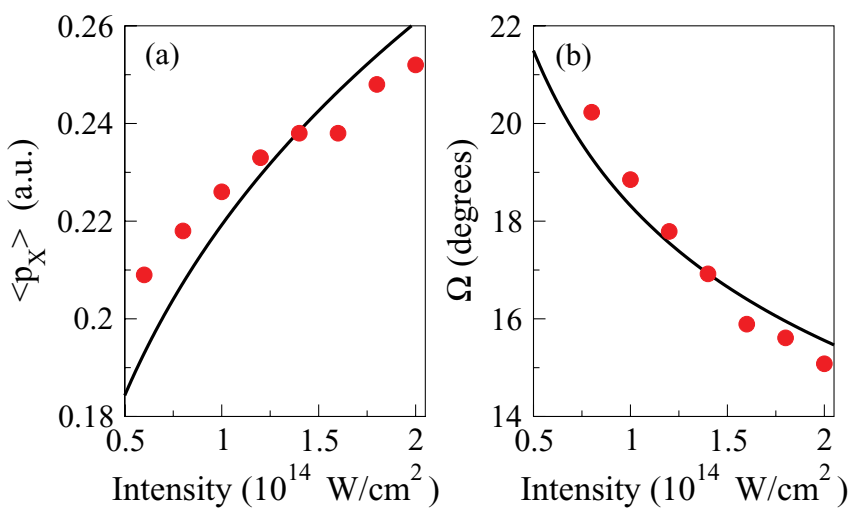

FIG. 8. (Color online) Expectation value of (a) $p_{X}$ and (b) off-nodal-plane emission angle $\Omega$ as a function of intensity of the circularly polarized field for a $p_{x}$ orbital with binding energy of 0.5 a.u. similar to the HOMO of benzonitrile and a laser wavelength of $800 \mathrm{~nm}$. The full curve denotes the present analytic theory, and the dots denote the results obtained from calculation within the strong-field approximation [42].

\section{STRONG-FIELD IONIZATION OF 3D-ORIENTED BENZONITRILE}

Armed with our generalized tunneling model including both the static Stark shifts and the off-the-nodal-plane emission, we now address the experimental results for benzonitrile.

As illustrated in Fig. 5, two sets of experiments have been performed involving preoriented benzonitrile molecules: one with 1D-oriented molecules and the other with 3D-oriented molecules. The results obtained with $1 \mathrm{D}$ and 3D orientation show almost the same degree of up-down asymmetry (Fig. 5). The asymmetry can be ascribed to the dependence of the ionization potential on the static Stark shifts of the energy levels (see Sec. IV A), i.e., the small difference between the dipole moment of benzonitrile and its cation (Appendix). The case of $1 \mathrm{D}$-oriented benzonitrile can be treated theoretically by the same method as introduced in Ref. [22] for the description of the linear OCS molecule. In the 1D-oriented case, the molecules are allowed to rotate freely around its molecular axis and the effect of off-the-nodal-plane emission is washed out. In the experiment on 3D-aligned and -oriented benzonitrile, on the other hand, the polarization plane of the circularly polarized laser lies entirely in the nodal plane of both HOMO and HOMO-1. Indeed, in this case, offthe-nodal-plane emission occurs and manifests itself as an $\mathrm{X}$-like pattern in the photoelectron momentum distribution on the detector screen, and here we focus on the 3D-oriented benzonitrile. To describe the experiment, we use the theory for off-the-nodal plane emission, developed in the previous section. The polarizability of benzonitrile is very large (see Appendix), so we expect that the initial orbital would be modified by the polarization response of the system, and we assume that any structure of the orbital off the nodal plane will be washed out. Thus, when the Stark shift is not included, the total probability of tunnel emission will not depend on the (finite) angle of the electric field vector with respect to the $Z$ axis. Therefore, we model all orbitals of benzonitrile that have their nodal plane coinciding with the polarization plane with the simplest possible orbital having such a nodal plane: the $p_{x}$ orbital. All theoretical results presented here assume
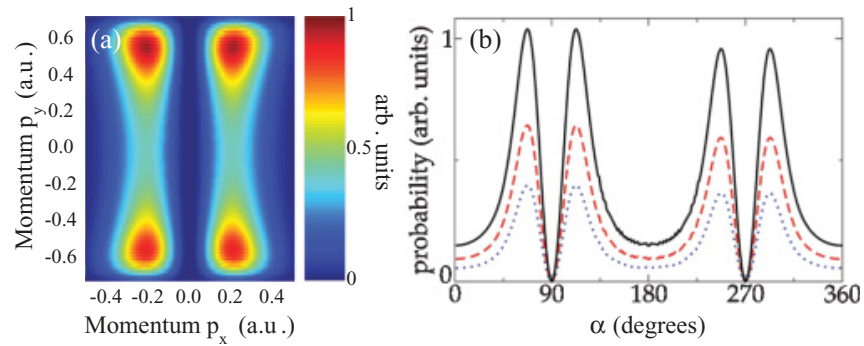

FIG. 9. (Color online) (a) Momentum distributions including volume effects for benzonitrile at $1.23 \times 10^{14} \mathrm{~W} / \mathrm{cm}^{2}$ for ionization from the HOMO and HOMO-1. (b) Angular distributions derived from momentum distributions. (Black solid) HOMO + HOMO-1; (red dashed) HOMO; (blue dotted) HOMO-1. The results should be compared to the corresponding experimental findings in Fig. 5.

perfect 3D orientation of the molecule. Using Eqs. (4), (7), and (9), the momentum distributions at a particular intensity can be calculated. We perform focal volume integration by including intensities ranging from $1.2 \times 10^{14}$ to $0.5 \times$ $10^{14} \mathrm{~W} / \mathrm{cm}^{2}$ in steps of $5 \times 10^{12} \mathrm{~W} / \mathrm{cm}^{2}$. The resulting momentum distributions from HOMO, HOMO-1, and the combined response of HOMO and HOMO-1 are shown in Fig. 9.

As seen by comparison of Figs. 5 and 9, at larger radial final momenta $p_{\rho}=\sqrt{p_{X}^{2}+p_{Y}^{2}}$, there is good agreement between the experimental and theoretical differential-momentum distributions. Also, the agreement between theory and experiment for the angle off-the-nodal-plane emission $\Omega$ is good: The experiment yields $\sim 18^{\circ}$ (Fig. 5, [Eq. (1)]) and the theory $18.8^{\circ}$ [Eq. (11)]. In agreement with the experiment the suppression of electron emission occurs at $\alpha=90^{\circ}$ and $270^{\circ}$. The degree of suppression seen experimentally is, however, much less pronounced than the complete suppression, i.e., no electron emission with $p_{X}=0$, predicted theoretically. One factor contributing to this disagreement is the lack of perfect alignment of the molecular plane in the experiment. Another source that can produce a signal at $p_{X}=0$ is emission from the HOMO-2 that is bound with additional 0.1 a.u. compared to the HOMO. The HOMO-2 has population in the polarization plane of the field so that its contribution could be nonvanishing (see Fig. 4 in the Supplementary Information of Ref. [22]).

The present theory is capable of describing the small up-total asymmetry observed in the experiment by attributing it to the Stark shift of the ionization potential due to the dipole and polarizability of the HOMO orbital, i.e., the difference between the permanent dipole moment and the polarizability of the molecule and those of the unrelaxed cation. The calculated up-total asymmetry for the case of a 3D-oriented molecule at $1.23 \times 10^{14} \mathrm{~W} / \mathrm{cm}^{2}$ is 0.52 in fair agreement with the experimental results $(0.55)$.

\section{CONCLUSIONS}

We have studied MFPADs following strong-field single ionization, with circularly polarized, nonresonant femtosecond pulses, of benzonitrile molecules aligned and oriented in space by mixed laser and static electric fields. The most striking experimental observation is suppression of electron emission in directions that correspond to nodal planes of the orbitals from which ionization occurs. The experimental findings are 
explained using a modified tunneling theory. Our theoretical analysis shows that the circularly polarized ionizing field drives the photoelectrons away from the cation, minimizing effects of postionization interaction, and leads to a clear signature of the orbital structure of the molecule at the time of ionization. In particular, we have shown here that nodal structures can be mapped out, a possibility recently examined further theoretically [54].

An important future challenge is to extend the present work to the study of time-dependent phenomena. This may be realized, for instance, by a pump-probe scheme where the pump pulse promotes molecules from the electronic ground state to an electronically excited state. One class of such photoinduced reactions that could be well suited for the strong-field probing, introduced here, concerns large amplitude vibrational motion in the excited state. This occurs for, instance, in axially chiral systems such as biphenyl or phenyl-pyrrole [55] where the dynamics in the excited states corresponds to torsional motion of two aromatic rings. Alignment as well as orientation of such systems is feasible [56,57] and the present probe method could map out the changing valence electronic structure during torsional motion occurs. More generally, strong-field ionization in combination with MFPADs may provide an informative probe of charge migration and charge transfer on time scales down to a few femtoseconds [58].

\section{ACKNOWLEDGMENT}

The work was supported by the Danish National Research Foundation, the Lundbeck Foundation, the Carlsberg Founda- tion, the Danish Council for Independent Research (Natural Sciences), and the European Marie Curie Initial Training Network Grant No. CA-ITN-214962-FASTQUAST.

\section{APPENDIX}

The molecular structure, population analysis, and orbital energies of benzonitrile were obtained from Hartree-Fock calculations [47] (the molecule is oriented in the $Y, Z$ plane and the $\mathrm{CN}$ end pointing in the positive $Z$ axis, see Fig. 5). The HOMO and HOMO-1 of benzonitrile are shown in Fig. 7. The orbital energies, given in Fig. 7, agree well with the experimental ionization potentials, reported in Ref. [59], and are very close in energy $\left[I_{p}: 9.79 \mathrm{eV}\right.$ (0.36 a.u) for HOMO; $10.06 \mathrm{eV}$ (0.37 a.u) for HOMO-1]. Population analysis shows that while the HOMO-1 is localized on the aromatic ring, about $30 \%$ of the HOMO electron density is on the $\mathrm{CN}$ end; see Fig. 7. The experimental 3D orientation of the molecule is such that the nodal planes of both HOMO and HOMO-1 orbitals coincide with the molecular plane, i.e., they have no electron density in the molecular plane.

For the purpose of our Stark-shift tunneling model we require the permanent dipole moment and the polarizabilities of benzonitrile and its cation. The nonzero elements of the polarizability tensor of benzonitrile are (in a.u.): $\alpha_{\mathrm{XX}}^{\mathrm{mol}}=50.522, \alpha_{\mathrm{YY}}^{\mathrm{mol}}=87.808, \alpha_{\mathrm{ZZ}}^{\mathrm{mol}}=125.758, \alpha_{\mathrm{XX}}^{\text {ion }}=$ $42.397, \alpha_{\mathrm{YY}}^{\text {ion }}=76.282$, and $\alpha_{\mathrm{ZZ}}^{\text {ion }}=151.467$, and the permanent dipole moment of benzonitrile is 1.791 a.u. (in fair agreement with the experimental value [60]) and 1.822 a.u. for the cation, both pointing along $-Z$ from the detector to the repeller.
[1] K. Reid, Annu. Rev. Phys. Chem. 54, 397 (2003).

[2] A. Stolow, A. E. Bragg, and D. M. Neumark, Chem. Rev. 104, 1719 (2004).

[3] A. Stolow and J. G. Underwood, Adv. Chem. Phys. 139, 497 (2008).

[4] P. Hockett, M. Staniforth, K. L. Reid, and D. Townsend, Phys. Rev. Lett. 102, 253002 (2009).

[5] T. Suzuki, Annu. Rev. Phys. Chem. 57, 555 (2006).

[6] E. Shigemasa, J. Adachi, M. Oura, and A. Yagishita, Phys. Rev. Lett. 74, 359 (1995).

[7] D. Akoury et al., Science 318, 949 (2007).

[8] M. S. Schöffler et al., Science 320, 920 (2008).

[9] M. Yamazaki, J. Adachi, T. Teramoto, A. Yagishita, M. Stener, and P. Decleva, J. Phys. B 42, 051001 (2009).

[10] J. A. Davies, R. E. Continetti, D. W. Chandler, and C. C. Hayden, Phys. Rev. Lett. 84, 5983 (2000).

[11] A. M. Rijs, M. H. M. Janssen, E. H. Chrysostom, and C. C. Hayden, Phys. Rev. Lett. 92, 123002 (2004).

[12] O. Gessner et al., Science 311, 219 (2006).

[13] Y. Tang, Y.-I. Suzuki, T. Horio, and T. Suzuki, Phys. Rev. Lett. 104, 073002 (2010).

[14] H. Stapelfeldt and T. Seideman, Rev. Mod. Phys. 75, 543 (2003).

[15] M. Meckel et al., Science 320, 1478 (2008).
[16] V. Kumarappan, L. Holmegaard, C. Martiny, C. B. Madsen, T. K. Kjeldsen, S. S. Viftrup, L. B. Madsen, and H. Stapelfeldt, Phys. Rev. Lett. 100, 093006 (2008).

[17] C. Z. Bisgaard, O. J. Clarkin, G. Wu, A. M. D. Lee, O. Gessner, C. C. Hayden, and A. Stolow, Science 323, 1464 (2009).

[18] B. Friedrich and D. Herschbach, J. Chem. Phys. 111, 6157 (1999).

[19] H. Sakai, S. Minemoto, H. Nanjo, H. Tanji, and T. Suzuki, Phys. Rev. Lett. 90, 083001 (2003).

[20] O. Ghafur, A. Rouzee, A. Gijsbertsen, W. K. Siu, S. Stolte, and M. J. J. Vrakking, Nat. Phys. 5, 289 (2009).

[21] L. Holmegaard, J. H. Nielsen, I. Nevo, H. Stapelfeldt, F. Filsinger, J. Küpper, and G. Meijer, Phys. Rev. Lett. 102, 023001 (2009).

[22] L. Holmegaard et al., Nat. Phys. 6, 428 (2010).

[23] D. Dimitrovski, M. Abu-samha, L. B. Madsen, F. Filsinger, G. Meijer, J. Küpper, L. Holmegaard, L. Kalhøj, J. H. Nielsen, and H. Stapelfeldt, Phys. Rev. A 83, 023405 (2011).

[24] T. K. Kjeldsen, C. Z. Bisgaard, L. B. Madsen, and H. Stapelfeldt, Phys. Rev. A 68, 063407 (2003).

[25] T. K. Kjeldsen, C. Z. Bisgaard, L. B. Madsen, and H. Stapelfeldt, Phys. Rev. A 71, 013418 (2005).

[26] U. Even, J. Jortner, D. Noy, N. Lavie, and C. Cossart-Magos, J. Chem. Phys. 112, 8068 (2000). 
[27] M. Hillenkamp, S. Keinan, and U. Even, J. Chem. Phys. 118, 8699 (2003).

[28] V. Kumarappan, C. Z. Bisgaard, S. S. Viftrup, L. Holmegaard, and H. Stapelfeldt, J. Chem. Phys. 125, 194309 (2006).

[29] F. Filsinger, J. Küpper, G. Meijer, L. Holmegaard, J. H. Nielsen, I. Nevo, J. L. Hansen, and H. Stapelfeldt, J. Chem. Phys. 131, 064309 (2009).

[30] N. F. Ramsey, Molecular Beams, The International Series of Monographs on Physics (Oxford University Press, London, 1956).

[31] K. Wohlfart, F. Filsinger, F. Grätz, J. Küpper, and G. Meijer, Phys. Rev. A 78, 033421 (2008)

[32] M. Kirste, B. G. Sartakov, M. Schnell, and G. Meijer, Phys. Rev. A 79, 051401 (2009).

[33] T. E. Wall, S. K. Tokunaga, E. A. Hinds, and M. R. Tarbutt, Phys. Rev. A 81, 033414 (2010).

[34] A. T. J. B. Eppink and D. H. Parker, Rev. Sci. Instrum. 68, 3477 (1997).

[35] J. J. Larsen, H. Sakai, C. P. Safvan, I. Wendt-Larsen, and H. Stapelfeldt, J. Chem. Phys. 111, 7774 (1999).

[36] J. J. Larsen, K. Hald, N. Bjerre, H. Stapelfeldt, and T. Seideman, Phys. Rev. Lett. 85, 2470 (2000).

[37] H. Tanji, S. Minemoto, and H. Sakai, Phys. Rev. A 72, 063401 (2005).

[38] I. Nevo, L. Holmegaard, J. H. Nielsen, J. L. Hansen, H. Stapelfeldt, F. Filsinger, G. Meijer, and J. Küpper, Phys. Chem. Chem. Phys. 11, 9912 (2009).

[39] The highest kinetic $\mathrm{CN}^{+}$ions appear as two sets of angularly confined rings. In accordance with similar observations for Coulomb explosion of iodobenzene [35] and difluoroiodobenzene [40] we interpret the innermost ring as resulting from $\mathrm{CN}^{+}$ions when $\mathrm{BN}$ is doubly ionized by the probe pulse and fragments into an $\mathrm{CN}^{+}+\mathrm{C}_{6} \mathrm{H}_{5}{ }^{+}$ion pair, whereas the outermost ring results from $\mathrm{CN}^{+}$ions formed from triple ionization and fragmentation into an $\mathrm{CN}^{+}+\mathrm{C}_{6} \mathrm{H}_{5}{ }^{2+}$ ion pair. The value of $\left\langle\cos ^{2} \theta_{2 \mathrm{D}}\right\rangle$ is determined in the radial range corresponding to the outermost ring.

[40] S. S. Viftrup, V. Kumarappan, S. Trippel, H. Stapelfeldt, E. Hamilton, and T. Seideman, Phys. Rev. Lett. 99, 143602 (2007).
[41] M. Abu-Samha and L. B. Madsen, Phys. Rev. A 82, 043413 (2010).

[42] L. V. Keldysh, Zh. Eksp. Teor. Fiz. 47, 1945 (1964) [Sov. Phys. JETP 20, 1307 (1965)].

[43] M. V. Ammosov, N. B. Delone, and V. P. Krainov, Zh. Eksp. Teor. Fiz. 91, 2008 (1986) [Sov. Phys. JETP 64, 1191 (1986)].

[44] X. M. Tong and C. D. Lin, J. Phys. B 38, 2593 (2005).

[45] K. Staliunas, Phys. Rev. Lett. 81, 81 (1998).

[46] P. B. Corkum, Phys. Rev. Lett. 71, 1994 (1993).

[47] M. W. Schmidt et al., J. Comput. Chem. 14, 1347 (1993).

[48] A. M. Perelomov, V. S. Popov, and M. V. Terent'ev, Zh. Eksp. Teor. Fiz. 50, 1393 (1966) [Sov. Phys. JETP 23, 924 (1966)].

[49] X. M. Tong, Z. X. Zhao, and C. D. Lin, Phys. Rev. A 66, 033402 (2002).

[50] C. Z. Bisgaard and L. B. Madsen, Am. J. Phys. 72, 249 (2004).

[51] N. B. Delone and V. P. Krainov, J. Opt. Soc. Am. B 8, 1207 (1991).

[52] T. Brabec, M. Côté, P. Boulanger, and L. Ramunno, Phys. Rev. Lett. 95, 073001 (2005).

[53] G. F. Gribakin and M. Y. Kuchiev, Phys. Rev. A 55, 3760 (1997).

[54] C. P. J. Martiny, M. Abu-samha, and L. B. Madsen, Phys. Rev. A 81, 063418 (2010).

[55] K. Okuyama, Y. Numata, S. Odawara, and I. Suzuka, J. Chem. Phys. 109, 7185 (1998).

[56] C. B. Madsen, L. B. Madsen, S. S. Viftrup, M. P. Johansson, T. B. Poulsen, L. Holmegaard, V. Kumarappan, K. A. Jørgensen, and H. Stapelfeldt, Phys. Rev. Lett. 102, 073007 (2009).

[57] C. B. Madsen, L. B. Madsen, S. S. Viftrup, M. P. Johansson, T. B. Poulsen, L. Holmegaard, V. Kumarappan, K. A. Jørgensen, and H. Stapelfeldt, J. Chem. Phys. 130, 234310 (2009).

[58] A. I. Kuleff and L. S. Cederbaum, Chem. Phys. 338, 320 (2007).

[59] T. Kobayashi and S. Nakagura, Bull. Chem. Soc. Jpn. 47, 2563 (1974).

[60] K. Wohlfart, M. Schnell, J. Grabow, and J. Küpper, J. Mol. Spectrosc. 247, 119 (2008). 